\title{
Sensitivity Analysis and Uncertainty in Linear Programming
}

\author{
Julia L. Higle • Stein W. Wallace \\ Department of Systems and Industrial Engineering, University of Arizona, Tucson, Arizona 85721 \\ Molde University College, PO Box 2110, N-6402 Molde, Norway \\ julie@sie.arizona.edu • stein.w.wallace@himolde.no
}

This paper was refereed.

\begin{abstract}
Linear programming (LP) is one of the great successes to emerge from operations research and management science. It is well developed and widely used. LP problems in practice are often based on numerical data that represent rough approximations of quantities that are inherently difficult to estimate. Because of this, most LP-based studies include a postoptimality investigation of how a change in the data changes the solution. Researchers routinely undertake this type of sensitivity analysis (SA), and most commercial packages for solving linear programs include the results of such an analysis as part of the standard output report. SA has shortcomings that run contrary to conventional wisdom. Alternate models address these shortcomings.

(Philosophy of modeling. Programming: stochastic.)
\end{abstract}

$\mathrm{L}$ inear programming (LP) has played an important role as a problem solving and analysis tool. Researchers have addressed a variety of important problems through linear programming. LP has been widely accepted and used for several reasons: First, it is taught in many educational settings. Students in engineering, business, and mathematics study the subject at some level, in some cases at the high school level! In addition, high quality software is available to assist researchers conducting LP-based investigations in building models, solving problems, and analyzing output.

Most authors of textbooks on LP discuss the need for sensitivity analysis (SA). In analyzing output, researchers use SA to explore how changes in the problem data might change the solution to a linear program, for example, how a change in production costs or demand projections might affect a production schedule. Because large-scale planning efforts often rely on large amounts of data, much of which represents best-guess estimates, the ability to undertake such sensitivity analyses is critical to the acceptance of the methodology. Indeed, people who are uncertain about data elements are often advised to use SA to resolve the impact of uncertainty. The use of SA to allay concerns about uncertainty draws attention to an issue that rarely arises in the development of LP models. While LP models often include time periods, they are typically the times at which decisions take effect (for example, production levels in a particular month). LP models generally do not reflect the times at which decisions are made. Nor do they distinguish between what will be known, and what will remain uncertain when the decisions are made. This lack of distinction derives from the history of LP's use primarily for deterministic problem solving. However, in planning under uncertainty, it is critical to properly reflect the manner in which decisions and information are interspersed. Typically, LP models do not offer such a reflection. As a consequence, the results of sensitivity analyses can be misleading.

\section{A Simple Example}

Our example is a variation of a problem described by Winston (1995):

The Dakota Furniture Company manufactures desks, tables, and chairs. A desk sells for $\$ 60$, a table 
HIGLE AND WALLACE

Sensitivity Analysis

\begin{tabular}{llrcc} 
& & \multicolumn{3}{c}{ Production Requirements } \\
\cline { 3 - 5 } Resource & Cost (\$) & Desk & Table & Chair \\
\hline Lumber (board feet) & 2 & 8 & 6 & 1 \\
Carpentry (hours) & 5.2 & 2 & 1.5 & 0.5 \\
Finishing (hours) & 4 & 4 & 2 & 1.5 \\
Demand & & 150 & 125 & 300
\end{tabular}

Table 1: Dakota requires lumber and labor (carpentry and finishing) to produce its products (desks, tables, and chairs). The cost of these resources varies. Resource requirements vary for each product.

sells for $\$ 40$, and a chair sells for $\$ 10$. The manufacture of each type of furniture requires lumber and two types of skilled labor: Carpentry and finishing (Table 1).

We can determine how much of each item to produce and the resources required to meet this production in a number of ways. Perhaps the easiest method is a simple per-item profit analysis. A desk costs $\$ 42.40$ to produce and sells for $\$ 60$, for a net profit of $\$ 17.60$. A table costs $\$ 27.80$ to produce and sells for $\$ 40$, for a net profit of $\$ 12.20$. That is, desks and tables are profitable. In the absence of constraints on resource availability, to maximize profit Dakota should produce as many of these items as it can sell (150 desks and 125 chairs). On the other hand, a chair costs $\$ 10.60$ to produce and sells for $\$ 10.00$, for a net loss of $\$ 0.60$. Based on the information provided, to maximize profit Dakota should stop producing chairs.

To produce 150 desks and 125 tables, Dakota needs:

$-1,950$ board feet of lumber,

- 487.5 hours of labor for carpentry,

-850 hours of labor for finishing, and it anticipates a profit of $\$ 4,165$ from selling the 150 desks and 125 tables.

At this point, we should review the method of analysis. In reality, Dakota must settle three issues:

- How much of each resource should it acquire?

- How many of each item should it produce?

- How many of each item should it sell?

The model behind our analysis does not consider these issues separately. Given the data in Table 1, we draw correspondences between these three issues and ensure that we produce only those items we can sell and acquire only the resources we need to produce them (Figure 1). Our model and analysis exploit the

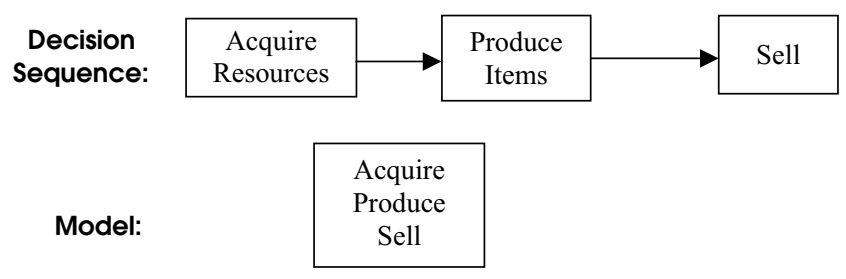

Figure 1: Dakota is actually faced with a sequence of three related decisions: How much resources to acquire, how many items to produce, and how many items to sell. The model represents these decisions as being made simultaneously, not sequentially.

structural advantages that accompany deterministic data and avoid representing potentially costly errors. In reality, the decisions occur sequentially over time.

This textbook problem is pretty straightforward. We do not need LP to solve it. However, for more complicated problems, an LP model is indispensable, so we describe one that considers each of the three decisions explicitly. In the following, let

$y_{d}=$ number of desks to produce,

$y_{t}=$ number of tables to produce,

$y_{c}=$ number of chairs to produce,

$x_{l}=$ number of board feet of lumber to acquire,

$x_{f}=$ number of labor hours to acquire for finishing,

$x_{c}=$ number of labor hours to acquire for carpentry,

$s_{d}=$ number of desks to sell,

$s_{t}=$ number of tables to sell, and

$s_{c}=$ number of chairs to sell.

With these variables, we can formulate Dakota's problem with the following LP:

Maximize $\quad-2 x_{l}-5.2 x_{c}-4 x_{f}+60 s_{d}+40 s_{t}+10 s_{c}$ (P.0)

subject to

$$
\begin{array}{rlrl}
-x_{l} & +8 y_{d}+6 y_{t}+y_{c} & \leq 0, \\
-x_{c} \quad+2 y_{d}+1.5 y_{t}+0.5 y_{c} & \leq 0, \\
-x_{f}+4 y_{d}+2 y_{t}+1.5 y_{c} & \leq 0, \\
s_{d} & & \leq 150, \\
s_{d} & -y_{d} & \leq 0, \\
s_{t} & & \leq 125, \\
s_{t} & \leq y_{t} & \leq 0, \\
s_{c} & \leq 300, \\
s_{c} & \leq 0, y_{c} & \leq 0, \\
x_{l}, x_{f}, x_{c}, y_{d}, y_{t}, y_{c}, s_{d}, s_{t}, s_{c} & \geq 0 .
\end{array}
$$

If the data in Table 1 changes, the structure of the model remains the same. For example, if Dakota 
raises the selling price of chairs from $\$ 10$ to $\$ 11$, we need only to change the corresponding coefficient in the objective function. This observation gives rise to the investigation of the solution known as SA. Knowing that the structure of the problem does not change, we can investigate how changes in individual data elements change the optimal solution. If nothing else changes when we increase the price of chairs from $\$ 10$ to $\$ 11$, producing chairs becomes profitable, and the nature of the solution changes considerably. On the other hand, if the selling price of chairs remains $\$ 10$, and the demand for chairs drops from 300 to 200, there would be no impact on the solution-Dakota would still not produce chairs.

Researchers use SA to study the robustness of solutions to LP models. That is, if they are concerned about the accuracy of the data, they perform SA to see how the solution might change if the data were different. A change in the solution or its structure would indicate the need for further investigation. When neither changes, they consider the proposed solution an appropriate guide for making the decision. The sense of security they gain from SA, however, is not well founded. Even when the solution and its structure appear to be stable, the proposed solution may be inappropriate in the face of uncertainty.

\section{Uncertainty in LP Data}

Demand for products may be uncertain, but low, most likely, and high values may be available. We will assume that the low values of demand for desks, tables, and chairs (50,20, and 200) occur with probability $p_{l}=0.3$, the most likely values $(150,110$, and 225 ) occur with probability $p_{m}=0.4$, and the high values $\left(250,250\right.$, and 500) will occur with probability $p_{h}=$ 0.3 . The possible demand scenarios and the corresponding probabilities form a distribution that we can use to describe future demand. The demand scenario presented in Table 1 is the expected value associated with the distribution in Table 2.

Analysis of the sensitivity of the solution to (P.0) indicates that our solution, "produce as many desks and tables as can be sold, but do not produce any chairs" will remain valid for any set of (nonnegative)

\begin{tabular}{lccc} 
Item & Low Value & Most Likely Value & High Value \\
\hline Desks & 50 & 150 & 250 \\
Tables & 20 & 110 & 250 \\
Chairs & 200 & 225 & 500 \\
Probability & 0.3 & 0.4 & 0.3
\end{tabular}

Table 2: Dakota is faced with three possible demand scenarios: Low demand values, most likely demand values, and high demand values. Each of these scenarios is modeled with specified demand for each product, and occurs with specified probabilities.

demands. Table 3 shows the optimal response to each of the individual demand scenarios.

In all cases, we produce only desks and tables, not chairs. We acquire resources to satisfy the production schedule. The production and resource quantities in the expected-value column are the expected values of the corresponding quantities in the remaining columns. (This is a property of the simplicity of the example; in general, the expected value of the data does not correspond to the expected value of the solutions.) Given the stability of the structure of the solution and the relationship among the various solutions, we might think that the solution with the expected demand is an appropriate response for Dakota's problem.

However, if Dakota produces 150 desks and 125 tables, to meet the mean demand solution, it has a 30 percent chance of producing too many desks and a 70 percent chance of producing too many tables. If it produces 150 desks and 125 tables and the

\begin{tabular}{lcrrr} 
& \multicolumn{4}{c}{ Demand } \\
\cline { 2 - 5 } Variables & Expected Value & Low & Most Likely & High \\
\hline Production quantities & & & & \\
$\quad$ Desks & 150 & 50 & 150 & 250 \\
Tables & 125 & 20 & 110 & 250 \\
$\quad$ Chairs & 0 & 0 & 0 & 0 \\
Resource quantities & & & & \\
$\quad$ Lumber (board feet) & 1,950 & 520 & 1,860 & 3,500 \\
Finishing (hours) & 850 & 240 & 820 & 1,500 \\
$\quad$ Carpentry (hours) & 487.50 & 130 & 465 & 875 \\
Profit (\$) & 4,165 & 1,124 & 3,982 & 7,450
\end{tabular}

Table 3: Each demand scenario that Dakota considers corresponds to an optimal solution. Production quantities and the resource acquisitions vary widely across the scenarios. 
low-demand scenario occurs (50 desks and 20 chairs), Dakota's profit will be much lower than $\$ 4,165$. The costs for resources at this level are $\$ 9,835$. Selling 50 desks and 20 chairs would bring in revenue of only $\$ 3,800$ for a net loss of $\$ 6,035$. If Dakota produced 150 desks and 125 tables and experienced the most likely demand, its net gain would be $\$ 3,565$. Although not a loss, this amount is well below the projected profit of $\$ 4,165$ suggested by the original LP solution.

No matter how we look at it, the analysis is flawed. If a firm bases its production on uncertain data, how great a potential error does it face? This may seem like a question SA can answer. In reality, a confusion of perspectives is at work. The LP model incorporates a kind of tunnel vision: For particular data, it tells what to do. The error analysis requires a broader view, a comparison of the manner in which the output associated with one set of data will perform if faced with something different. SA does not address this issue.

\section{LP Models with Uncertainty}

When faced with uncertainty in the demand for products, we need a more thoughtful approach to model development. In this case, we need to capture the relationship between the times at which we will make decisions and the time at which we will know the demand. We can adapt decisions made after the demand is known to the specific demand scenariosomething we cannot do for decisions made before we know the demand. To provide a proper forum for assessing the trade-offs among the various alternatives, we need a model that captures the flexibility the decision process affords. Logically, three potential information timings are of concern (Figure 2).

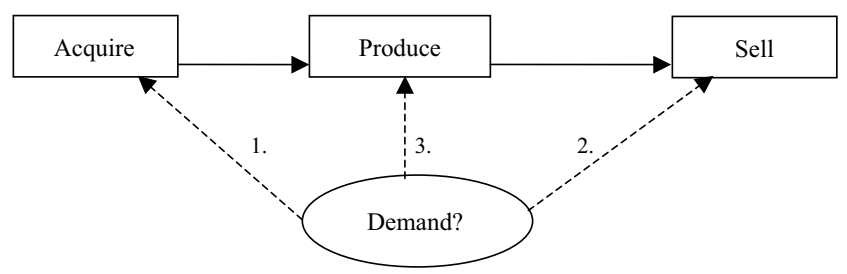

Figure 2: When will demand be known? When demand is uncertain, it is important to know when it will be revealed to the decision maker. Will it be known before resources are acquired, between acquisition and production, or after production decisions are made?
That is, we should determine the point during the decision sequence at which we know the demand. We might have complete information about the demand before making any decisions. At the other extreme, we might not know the demand until after we acquire resources and produce items. The demand determines the actual sales quantities and consequently our revenues. An intermediate possibility is that we acquire resources while we are uncertain about the demand, but we set the production schedules only after we know the demand and thus have adapted to it.

These three possibilities give rise to three different types of models. In the first case, we know demand at the start and can base decisions about acquiring resources, production, and sales on whether demand is low, most likely, or high (Figure 3).

If demand is known at the start, our decisions are not exposed to uncertainty, and we need no cross-scenario evaluation. Because all uncertainty is resolved before we make any decisions, we adapt any decision to the specific scenario realized, and the problem collapses into a collection of deterministic problems; only the origin remains uncertain. To formulate this problem, we need three separate sets of variables, one for each possible demand scenario (low, most likely, high). An LP model for this problem will be separable by scenario. Working from (P.0), and letting $D_{d s}$ denote the demand for desks under scenario $s$ (with $D_{t s}$ and $D_{c s}$ similarly defined), we obtain

$$
\sum_{\{s \in\{l, m, h\}\}}^{\text {Maximize }}\left(-2 x_{l s}-5.2 x_{c s}-4 x_{f s}+60 s_{d s}+40 s_{t s}+10 s_{c s}\right) p_{s}
$$

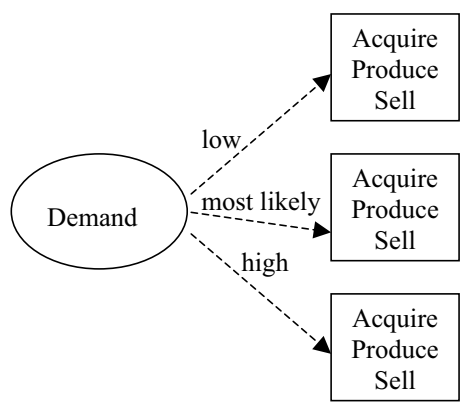

Figure 3: If demand will be known before any decision is made, the decision tree contains the deterministic model depicted in Figure 1. It is replicated for each demand scenario. 
subject to

$$
\begin{aligned}
& -x_{l s}+8 y_{d s}+6 y_{t s}+y_{c s} \leq 0, \quad s \in\{l, m, h\}, \\
& -x_{c s}+2 y_{d s}+1.5 y_{t s}+0.5 y_{c s} \leq 0, \quad s \in\{l, m, h\}, \\
& -x_{f s}+4 y_{d s}+2 y_{t s}+1.5 y_{c s} \leq 0, \quad s \in\{l, m, h\}, \\
& s_{d s} \quad \leq D_{d s}, s \in\{l, m, h\}, \\
& s_{d s} \quad-y_{d s} \quad \leq 0, \quad s \in\{l, m, h\}, \\
& s_{t s} \quad \leq D_{t s}, s \in\{l, m, h\}, \\
& s_{t s} \quad-y_{t s} \quad \leq 0, \quad s \in\{l, m, h\}, \\
& s_{c s} \quad \leq D_{c s}, s \in\{l, m, h\}, \\
& s_{c s} \quad-y_{c s} \leq 0, \quad s \in\{l, m, h\}, \\
& x_{l s}, x_{f s}, x_{c s}, y_{d s}, y_{t s}, y_{c s}, s_{d s}, s_{t s}, s_{c s} \geq 0, \quad s \in\{l, m, h\} \text {. }
\end{aligned}
$$

As indicated, (P.1) is separable by scenario. We can consider each demand scenario separately, and we can obtain scenario-specific solutions independently. Only in calculating the objective value do we combine them. At the other extreme, we determine both acquisition and production before we know the demand (2 in Figure 2) (Figure 4).

Once made, the decisions about acquisition and production are fed into the demand uncertainty. Only the sales levels respond to the acquisition and production levels and the manner in which the demand uncertainty is resolved. Any LP model of this problem must capture the fact that the initial decisions must be weighed against all possible demand scenarios. To accomplish this, we use three separate sets of the sell variables, and only one set of the acquisition and production variables. As before, we work from (P.0) to develop our model. To connect Figure 4 and the LP

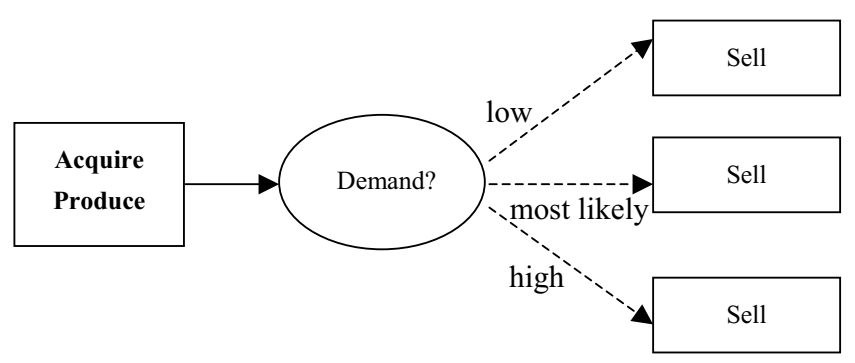

Figure 4: If demand is known after acquisition and production are determined, it will affect only the amount of product that is sold. model, we use a bold font to identify decisions made before demand is known.

Maximize

$-2 \mathbf{x}_{l}-5.2 \mathbf{x}_{c}-4 \mathbf{x}_{f}+\sum_{\{s \in\{l, m, h\}\}}\left(60 s_{d s}+40 s_{t s}+10 s_{c s}\right) p_{s}$

subject to

$$
\begin{aligned}
& -\mathbf{x}_{l}+8 \mathbf{y}_{d}+6 \mathbf{y}_{t}+\mathbf{y}_{c} \leq 0 \\
& -\mathbf{x}_{c}+2 \mathbf{y}_{d}+1.5 \mathbf{y}_{t}+0.5 \mathbf{y}_{c} \leq 0 \\
& -\mathbf{x}_{c}+4 \mathbf{y}_{d}+2 \mathbf{y}_{t}+1.5 \mathbf{y}_{c} \leq 0 \\
& s_{d s} \quad \leq 0, \quad s \in\{l, m, h\}, \\
& \mathbf{y}_{t} \\
& s_{t s} \leq D_{t s}, s \in\{l, m, h\} \\
& s_{t s} \quad \leq 0, \quad s \in\{l, m, h\}, \\
& s_{c s} \leq D_{c s}, s \in\{l, m, h\}, \\
& -\mathbf{y}_{c} \quad s_{c s} \leq 0, \quad s \in\{l, m, h\}, \\
& \mathbf{x}_{l}, \mathbf{x}_{f}, \mathbf{x}_{c}, \mathbf{y}_{d}, \mathbf{y}_{t}, \mathbf{y}_{c}, S_{d s}, S_{t s}, S_{c s} \geq 0, \quad s \in\{l, m, h\} .
\end{aligned}
$$

In contrast to (P.1), (P.2) is not separable by scenario. Acquisition and production, represented by $\mathbf{x}$ and $\mathbf{y}$, are determined before demand is known and are held constant across all scenarios. The second set of constraints models the manner in which sales depend on the combination of production and demand. The lack of separability arises because of the interaction of the two types of variables in these constraints.

Finally, in the remaining case (3 in Figure 2), we determine acquisition before we know the demand and production and sales afterward (Figure 5).

As we work from (P.0) to develop an LP model for this problem, we have a single set of acquisition variables, and three sets of production and sales variables:

Maximize

$-2 \mathbf{x}_{l}-5.2 \mathbf{x}_{c}-4 \mathbf{x}_{f}+\sum_{\{s \in\{l, m, h\}\}}\left(60 s_{d s}+40 s_{t s}+10 s_{c s}\right) p_{s}$

subject to

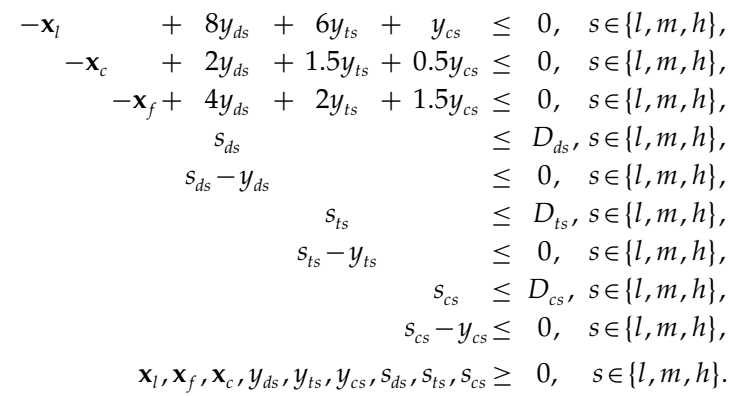

INTERFACES

Vol. 33, No. 4, July-August 2003 


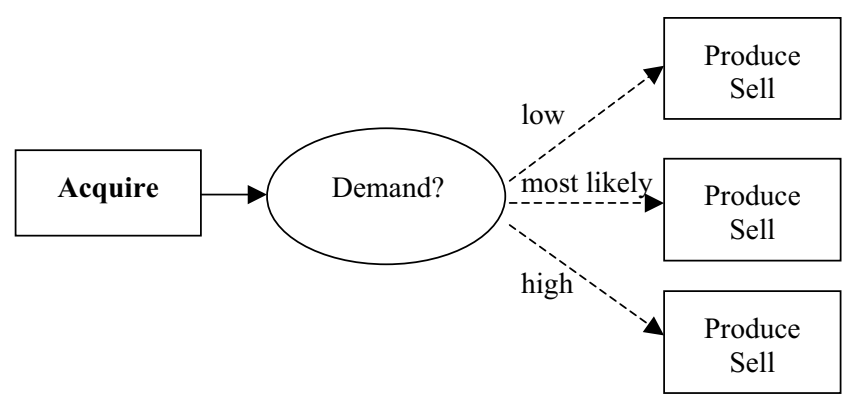

Figure 5: If demand will be known after resources are acquired but before production levels are determined, it will affect production quantities and sales.

Similar to (P.2), (P.3) lacks separability. In general, separability does not occur when the LP model includes uncertainty within the midst of the decision sequence.

\section{Comments on Problem Formulations and Solutions}

The three LP models, (P.1) through (P.3), can be traced back to the original model, (P.0), but they differ. They represent three different models of the problem. We have little need for a model such as (P.1). Because we know the demand before making any decisions, we do not need to solve (P.1). That is, we can wait until we know the demand and solve the appropriate scenario problem. As presented, the output of (P.1) provides the optimal solution and objective values for all possible demand scenarios. For planning, this information might be helpful.

The second model, (P.2), provides a proper mechanism for determining the expected revenues when we must determine production before we know the demand. This model accounts for the possibility that production might exceed demand. In particular, when we set production levels (which in turn determine the levels of resource acquired), we base them upon a model of the revenues that we can expect from selling them.

The third model, (P.3), separates acquisition from production. It is appropriate when we can make alternate production plans depending on demand that materializes from particular acquisitions. That is, it models the case in which the firm can use resources in a variety of ways to create products for which there is demand. To further appreciate the differences among the three models, we can compare their output (Table 4).

Although the output to (P.2) is structurally similar to that of the individual scenario problems in (P.1), the values are different. In (P.2), the firm produces items prior to knowing the demand. Unlike (P.1), the pro-

\begin{tabular}{|c|c|c|c|c|c|c|c|c|}
\hline \multirow[b]{2}{*}{ Variables } & \multirow{2}{*}{$\begin{array}{c}(P .0) \\
\text { Mean } \\
\text { Demand }\end{array}$} & \multicolumn{3}{|c|}{ (P.1) Scenarios } & \multirow[b]{2}{*}{$(\mathrm{P} .2)$} & \multirow{2}{*}{\multicolumn{2}{|c|}{ (P.3) }} & \\
\hline & & Low & Most Likely & High & & & & \\
\hline \multirow{6}{*}{$\begin{array}{l}\text { Lumber } \\
\text { Finishing labor } \\
\text { Carpentry labor }\end{array}$} & \multirow{6}{*}{$\begin{array}{l}1,950 \\
850 \\
487.5\end{array}$} & \multicolumn{3}{|c|}{ Resource Quantities } & \multirow{6}{*}{$\begin{array}{r}1,060 \\
420 \\
265\end{array}$} & \\
\hline & & 520 & 1,860 & 3,500 & & \multicolumn{3}{|c|}{1,300} \\
\hline & & 240 & 820 & 500 & & \multicolumn{3}{|c|}{540} \\
\hline & & 130 & 465 & 875 & & \multirow{2}{*}{\multicolumn{3}{|c|}{$\begin{array}{c}325 \\
\text { Demand }\end{array}$}} \\
\hline & & & & & & & & \\
\hline & & \multicolumn{3}{|c|}{ Production Quantities } & & Low & Most Likely & High \\
\hline Desks & 150 & 50 & 150 & 250 & 50 & 50 & 80 & 80 \\
\hline Tables & 125 & 20 & 110 & 250 & 110 & 20 & 110 & 110 \\
\hline Chairs & 0 & 0 & 0 & 0 & 0 & 200 & 0 & 0 \\
\hline Objective value & 4,165 & & 4,165 & & 1,142 & & 1,730 & \\
\hline
\end{tabular}

Table 4: Each of the problems (P.0) through (P.3) has a different optimal solution. The objective values differ as well, even when the structures of the optimal solutions are similar. 
duction levels suggested by (P.2) do not match any of the demand scenarios. In (P.2), production levels are set in a manner that balances the potential sunk cost of producing items that cannot be sold against the potential revenue available from selling a larger number of items. This balancing act shifts the production level away from any one scenario. We cannot recognize the need for this balance with a simple SA of the solution to (P.0). More important, the structure of the solution to (P.3), in which production decisions are delayed until after the demand is known, is distinctly different from the structures of the solutions to the other models. It is the only model that includes the production of chairs in the optimal solution and then only in the low-demand scenario. The interpretation of this solution is clear. Although chairs on their own are not profitable, their production in some cases is advantageous. The solution to (P.3) includes acquisition of a larger amount of resource than the solution to (P.2). When the demand is high enough, all of this resource goes toward the production of desks and chairs (the profitable items). However, when the demand is low, production of chairs offers the firm an opportunity to recoup much of the cost of the resources acquired. The chairs provide the firm with a fallback position that permits an aggressive resource acquisition plan. Again, we cannot realize the advantages of this adaptation with a simple SA of the solution to (P.0).

The various objective values differ as well. It is well known that solving an LP in which random variables in the right-hand sides of the constraints are replaced by their expected values yields an optimistic objective value, as indicated in (P.0) compared to the rest. Indeed, in this case, (P.0) is as optimistic as (P.1), in which the decision maker knows all information before making any decisions (although this need not be the case in general)! That the objective value for (P.3) exceeds that of (P.2) is no surprise; delaying decisions until one has information usually brings economic advantages. To determine the appropriate model, one must identify the point at which information about demand will be available.

\section{Alternate Objective Functions}

Our LP models have the objective of maximizing expected profit. There are many valid criticisms of

\begin{tabular}{lcccc} 
& & \multicolumn{3}{c}{ Profit Generated } \\
\cline { 3 - 5 } Demand & Probability & Alternative 1 & Alternative 2 & Alternative 3 \\
\hline Low & 0.3 & 0 & -100 & -300 \\
Most likely & 0.4 & 0 & 0 & -300 \\
High & 0.3 & 0 & -100 & 700
\end{tabular}

Table 5: The three alternatives yield different profits for each demand scenario. The expected value of the profits associated with all three alternatives is zero.

this choice of objective function. Because the expected value is a linear functional, gains and losses can cancel each other out. That is, suppose that we have three alternatives that yield profit distributions as a function of demand (Table 5).

Given the objective of maximizing expected profit, we would not distinguish between these three alternatives. This lack of distinction is inconsistent with most people's attitudes toward risk-most people have a clear preference among these three alternatives. The economist addresses this problem through utility theory, using a utility function that encapsulates the trade-off between expected profit and risk to guide the decision-making process. In general, optimization of expected utility requires a nonlinear objective function, although piecewise linear approximations can often be developed. In addition to the changes in the constraints, uncertainty may also result in a change in the objective function.

\section{Discussion}

Managing data when constructing LP models can be challenging. Planning models often address decisions to be made in the future. The farther into the future we project, the less certain we may be about prices, demands, supplies, and other quantities that typically appear in LP models. As a result, the data used in LP models is often clouded with uncertainty. For years, we have looked to SA to assure us of the quality of LP solutions when our data was uncertain. We should recognize that these assurances are not free. If we want to understand the impact of the uncertainty in data, we have to develop models that specifically incorporate uncertainty. In formulating such models, we must consider the relationships between the times 
at which decisions are made and the times at which we have certain data.

In most cases, the output of SA is misleading when used to assess the impact of uncertainty. SA is most appropriate when the basic structure of the model is not altered by the presence of uncertainty-for example, when all uncertainties will be resolved before any decisions are made. When the decisions are to be made, a deterministic model will be appropriate, but as long as the data is uncertain, we do not know which deterministic model will be appropriate. In this situation, SA can help us to appreciate the impact of uncertainty. In all other cases, we cannot count on it to do so.

SA fails as a tool for gauging the impact of uncertainty because it cannot capture the possibility of a response to information. When we obtain information during a decision sequence, we have the opportunity to adapt to it. Whether the adaptation is imposed, as when sales are constrained by demand, or advantageous, as when production decisions can be delayed until after demand is known, adaptation causes changes in the LP model. The constraint matrix changes considerably, affecting both the number of constraints and the number of variables. Because SA depends on an enduring structure in the LP model, it is not an appropriate tool for identifying the impact of uncertainty in these cases.

\section{Conclusion}

Under uncertainty, we cannot predict the conditions we will face tomorrow. A decision made today affects what we can do tomorrow. Similarly, what we ultimately decide tomorrow will depend on what we have learned today. Today's decision should be balanced against the conditions that we might face so that we can be reasonably confident about the position that we will be in tomorrow. When a model is based on the presumption of deterministic data, learning is absent in both the model and its output. SA based on the output of such a model will not reflect an ability to adapt to information that becomes available within a sequential decision process. It does not perform the balancing act required for decision making under uncertainty.

\section{Acknowledgment}

We gratefully acknowledge the Centre for Advanced Study at the Norwegian Academy of Science and Letters, whose support during the 2000-2001 academic year made this work possible.

\section{Reference}

Winston, W. L. 1995. Introduction to Mathematical Programming: Applications and Algorithms, 2nd ed. Duxbury Press, Belmont, CA. 